\title{
ARTICLE \\ Sequential light programs shape kale (Brassica napus) sprout appearance and alter metabolic and nutrient content
}

\author{
Sofia D Carvalho ${ }^{1,2}$ and Kevin M Folta ${ }^{1,2,3}$
}

Different light wavelengths have specific effects on plant growth and development. Narrow-bandwidth light-emitting diode (LED) lighting may be used to directionally manipulate size, color and metabolites in high-value fruits and vegetables. In this report, Red Russian kale (Brassica napus) seedlings were grown under specific light conditions and analyzed for photomorphogenic responses, pigment accumulation and nutraceutical content. The results showed that this genotype responds predictably to darkness, blue and red light, with suppression of hypocotyl elongation, development of pigments and changes in specific metabolites. However, these seedlings were relatively hypersensitive to far-red light, leading to uncharacteristically short hypocotyls and high pigment accumulation, even after growth under very low fluence rates $\left(<1 \mu \mathrm{mol} \mathrm{m} \mathrm{m}^{-2} \mathrm{~s}^{-1}\right)$. General antioxidant levels and aliphatic glucosinolates are elevated by far-red light treatments. Sequential treatments of darkness, blue light, red light and far-red light were applied throughout sprout development to alter final product quality. These results indicate that sequential treatment with narrow-bandwidth light may be used to affect key economically important traits in high-value crops.

Horticulture Research (2014) 1, 8; doi:10.1038/hortres.2014.8; published online 12 March 2014

\section{INTRODUCTION}

Light is not only used by plants for photosynthesis, it is also a potent driver of variation in growth, development, physiology and metabolism. Specific wavelengths of light provide discrete information to the plant leading to particular responses. For instance, blue light (400-500 nm) controls phototropic growth, leaf expansion, stem growth inhibition and accumulation of anthocyanin pigments. Red light $(\sim 660 \mathrm{~nm})$ controls many responses including germination, functions of the chloroplast, stem and petiole growth. Far-red light $(>700 \mathrm{~nm})$ is an important signal in a shaded environment, and has a central role in modulating red light responses. All of these light wavelengths have well-described effects on flowering and gene expression.

Brassica sprouts have been shown to be rich in many phytonutrients including anthocyanins, glucosinilates (GLs), sulforaphane, carotenoids, flavonoids, general antioxidants, and terpenes. ${ }^{1,2}$ Fahey et al. ${ }^{3}$ demonstrated that cruciferous sprouts had 10-100 times the quantity of chemoprotective compounds than mature plants. Qualitative and quantitative differences in phytonutrients are also observed during germination and seedling development. ${ }^{4}$ Some of these compounds have been shown to have potential anticancer properties, as demonstrated by in vitro assays ${ }^{5,6}$ and in mice. ${ }^{7}$ Consumption of broccoli sprouts is associated with a risk reduction in populations exposed to environmental pollutants. ${ }^{8}$ Many studies document the chemoprotective effects of compounds derived from sprouts, and clinical trials indicate that they may be consumed without any ill effects. ${ }^{9}$

Brassica sprouts possess an innate ability to produce an array of phytonutrients. Is it possible to alter the light environment to maximize this potential? It has been shown that secondary metabolite networks do change in response to environmental cues such as heat stress, ${ }^{10}$ nitrogen and sulfur availability ${ }^{11}$ and ultraviolet (UV)-B irradiation. ${ }^{12}$ Narrow-bandwidth light-emitting diodes
(LEDs) have been used to analyze changes in carotenoid and GL levels in mature kales ${ }^{13}$ and broccoli sprout nutrient levels could be increased with specific treatments of blue light. ${ }^{14}$ The objective of this work is to test the effect of narrow-bandwidth light treatments on shaping aspects of kale sprout growth, and then combining them into an optimized program that could be used to produce sprouts with enhanced qualities.

In this report, we use narrow-bandwidth light sources provided by custom LED arrays to develop, and then control, size, pigment accumulation and nutraceutical content in Red Russian Kale (Brassica napus L. subsp. napus var pabularia). Red Russian kale was chosen because it is gaining in popularity as a healthful food and also exhibited significant phenotypic plasticity in preliminary experiments. The results show that kale seedlings follow the general rules defined by Arabidopsis, with some exception in the response to far-red light. The seedlings demonstrate wavelengthdependent alterations in anthocyanin content, GLs and general antioxidant qualities. These findings demonstrate that a vocabulary of specific light treatment sequences may be applied to derive remarkably different outcomes from a single genetic background.

\section{MATERIALS AND METHODS}

Plant materials

Seeds for Red Russian kale (Brassica napus pabularia; Johnny's Selected Seeds, Waterville, ME, USA) were surface sterilized in $25 \%(\mathrm{v} / \mathrm{v})$ bleach for $10 \mathrm{~min}$ followed by a brief treatment with $70 \%$ ethanol and then were placed on vertical water-agar plates or on horizontal water-agar magenta boxes for the final sequential treatments. The seeds were stratified at $4{ }^{\circ} \mathrm{C}$ for $48 \mathrm{~h}$, exposed to white light for $1 \mathrm{~h}$ and transferred to darkness for 24-96 $\mathrm{h}$ prior to light treatments.

Light conditions

LED light was provided by Plant Whisperer light units (Light Emitting Computers, Victoria, BC, Canada). The wavelengths tested were 470, 660

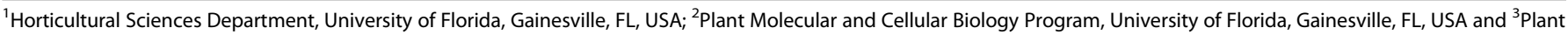
Innovation Program, University of Florida, Gainesville, FL, USA

Correspondence: KM Folta, Horticultural Sciences Department, 1301 Fifield Hall, University of Florida, Gainesville, FL 32611, USA (kfolta@ufl.edu)

Received: 31 October 2013; accepted: 17 December 2013
} 
and $730 \mathrm{~nm}$, along with white light supplied by Philips Cool White Fluorescent Bulbs (Newark, NJ). Experimental trials were conducted in ventilated experimental chambers lined with reflective mylar. Light was applied at various fluence rates, as stated in figures, without photoperiod.

Fluence rate response/developmental competence

Seeds on agar plates were transferred to complete darkness and then were moved to light conditions on sequential days. Seedlings were grown under various fluence rates and wavelengths, alone or in combination as described. End-point hypocotyl length was measured after $96 \mathrm{~h}$. Seedlings were imaged on a flat-bed scanner and then were measured using Image Tool 3.0 (Austin, TX).

Extraction and measurement of anthocyanins and chlorophyll Four-day-old seedlings grown under different light conditions were collected, roots-excised, immediately frozen in liquid nitrogen, reduced to powder using a mortar and a pestle, and transferred to an eppendorf tube where the weight was measured. Around $60 \mathrm{mg}$ and $20 \mathrm{mg}$ of powder weight were used for anthocyanins and for chlorophyll extraction, respectively.

Anthocyanins extraction followed the method described by Neff and Chory. ${ }^{15}$ Three hundred microliters of methanol-1\% (v/v) HCl were added to each tube and incubated overnight at $4{ }^{\circ} \mathrm{C}$ under dark. In total, $200 \mu \mathrm{L}$ of water and $500 \mu \mathrm{L}$ of chloroform were then added and the tubes were centrifuged for $5 \mathrm{~min}$ at maximum speed at room temperature. The supernatant was transferred to a new tube and the volume was adjusted to $800 \mu \mathrm{L}$ with $60 \%$ methanol- $1 \% \mathrm{HCl}$. The absorbances at $530 \mathrm{~nm}$ and $657 \mathrm{~nm}$ were read with a SmartSpec 3000 spectrophotometer (Bio-Rad, Hercules, (A), using $60 \%$ methanol-1\% $\mathrm{HCl}$ as the blank. Anthocyanins per seedling weight was calculated using the following equation:

$$
\text { Antho }=\left(\mathrm{Abs}_{530}-\mathrm{Abs}_{657}\right) \times 1000 \times \text { powder weight }(\mathrm{mg})^{-1}
$$

For chlorophyll extraction the method used was described. ${ }^{16}$ Ground leaf tissue is added to $800 \mu \mathrm{L}$ of dimethylformamide and incubated overnight at $4{ }^{\circ} \mathrm{C}$ in darkness. The next day, the absorbance was recorded at $647 \mathrm{~nm}$ and $664 \mathrm{~nm}$ in a quartz cuvette, using dimethylformamide as the blank. The total level of chlorophyll per seedling weight was calculated using the following equation:

$$
\mathrm{Ch}=0.8 \times\left(17.67 \times \mathrm{Abs}_{647}+7.17 \times \mathrm{Abs}_{664}\right) \times \text { powder weight }(\mathrm{mg})^{-1}
$$

Measurement of total antioxidant capacity

The antioxidant capacity was determined following the oxygen radical absorbance capacity-fluorescein (ORAC-FL) method described by Cao et $a .^{17}$ and modified by Ou et al. ${ }^{18}$. Four-day-old seedlings, grown for 1 day under darkness and 3 days under light at $50 \mu \mathrm{mol} \mathrm{m} \mathrm{m}^{-2} \mathrm{~s}^{-1}$ fluence rate, were collected, roots-excised and frozen in liquid nitrogen. The material was ground to powder and the weight was registered. Around $150 \mathrm{mg}$ were used per sample. Five milliliters of ice-cold phosphate-buffered saline $(\mathrm{pH}$ 7.0) were added to the powder and incubated for $1 \mathrm{~h}$ on ice, under dark. Each solution was centrifuged for $30 \mathrm{~min}$ at 4000 r.p.m. and $4{ }^{\circ} \mathrm{C}$, the supernatant transferred to three microcentrifuge tubes, and then centrifuged for $20 \mathrm{~min}$ at $20000 \mathrm{~g}$ and $4{ }^{\circ} \mathrm{C}$. The supernatants were collected again into three new tubes, used as three technical replicates in each independent experiment. These solutions were kept on ice and immediately used for antioxidant capacity analysis or frozen at $-80^{\circ} \mathrm{C}$.

The ORAC-FL was conducted at $37{ }^{\circ} \mathrm{C}$ in a $3 \mathrm{~mL}$ final volume (Phosphate Buffered Saline solvent). The fluorescence was recorded every $5 \mathrm{~min}$ in a FluoroMax-3 fluorometer (Jobin Yvon Horiba, Edison, NJ) using $480 \mathrm{~nm}$ and $514 \mathrm{~nm}$ as the excitation and emission wavelengths, respectively. The reactions were conducted for $90 \mathrm{~min}$ or until a reaction had stopped (considered when the decay in fluorescence would be lower than $5 \%$ of the previous reading). Next, $300 \mu \mathrm{L}$ of the diluted kale extracts were added to the cuvette containing fluorescein (sodium salt; Sigma, St. Louis, MO) to a final concentration of $100 \mathrm{nM}$ and equilibrated at $37^{\circ} \mathrm{C}$ for $15 \mathrm{~min}$. 2,2'-azobis(2-methylpropionamidine) dihydrochloride (Sigma) was then added to a final concentration of $12 \mathrm{mM}$ and the reaction started. To build the calibration curve, the Trolox reagent ( \pm-6 -hydroxy-2,5,7,8-tetramethylchromane-2carboxylic acid; Sigma) replaced the kale extracts in the reaction volume and the fluorescences of eight solutions with known Trolox concentrations from 1 to $8 \mu \mathrm{M}$ were measured. Fluorescein and 2,2'-azobis(2-methylpropionamidine) dihydrochloride constituted the blank.
The area under the fluorescence decay curve, $A U C$, in a plot of the relative (to time zero, $F L_{0}$ ) fluorescence over time, was calculated using the following equation, where $F L_{i}$ is the fluorescence level read at a time point $i$ and $\Delta t$ is the interval between reads ( $5 \mathrm{~min}$ in our experimental conditions):

$$
\mathrm{AUC}=\left(\mathrm{FL}_{0} / \mathrm{FL}_{0}+\mathrm{FL}_{1} / \mathrm{FL}_{0}+\mathrm{FL}_{2} / \mathrm{FL}_{0}+\ldots+\mathrm{FL}_{\mathrm{i}} / \mathrm{FL}_{0}\right) \times \Delta t(\min )
$$

The difference between the AUC for a given sample and the blank, $A U C_{\text {blank, }}$ gives the net area, Net $A U C$ :

$$
\text { Net } A U C=A U C-A U C_{\text {blank }}
$$

Plotting the Net AUC of the Trolox samples versus the known Trolox concentrations allowed building a calibration curve and to extrapolate a concentration of Trolox equivalents in the kale extracts.

\section{Measurement of GL content}

For GL extraction around $100 \mathrm{mg}$ of 4-day-old seedlings (roots-excised) were frozen in liquid nitrogen and reduced to powder in a microcentrifuge tube. One milliliter of $70 \%$ methanol at $80{ }^{\circ} \mathrm{C}$ and $30 \mu \mathrm{L}$ of $5 \mathrm{mM}$ benzylglucosinolate (glucotropaeolin potassium salt; ChromaDex, Irvine, CA) were added to each sample tube, vortexed and incubated at $80{ }^{\circ} \mathrm{C}$ for $10 \mathrm{~min}$. The tubes were centrifuged at $4000 \mathrm{~g}$ for $10 \mathrm{~min}$ (room temperature) and the supernatant transferred to a $15 \mathrm{~mL}$ round-bottomed test tube. This methanol extraction was repeated twice, the supernatants combined and the volume adjusted to $4 \mathrm{~mL}$ with $70 \%$ methanol.

For GL purification, $1 \mathrm{~g}$ DEAE sephadex A-25 resin (Sigma) was incubated overnight in $30 \mathrm{~mL} 0.5 \mathrm{M}$ acetate buffer ( $\mathrm{pH} 5.0$ ) and $1 \mathrm{~mL}$ was applied to a Bio-Rad column. The column was washed once with $5 \mathrm{~mL}$ water, the $\mathrm{GL}$ extract was then added, and the column was washed again twice with $2 \mathrm{~mL} \mathrm{70 \%} \mathrm{methanol,} \mathrm{five} \mathrm{times} \mathrm{with} 2 \mathrm{~mL}$ water and once with $2 \mathrm{~mL}$ $20 \mathrm{mM}$ acetate buffer. Fifteen milligram of sulfatase (Sigma) were dissolved in $6 \mathrm{ml} 20 \mathrm{mM}$ acetate buffer and $500 \mu \mathrm{L}$ was applied to each column and then left overnight at room temperature. The columns were eluted three times with $1 \mathrm{~mL}$ water, the eluted fractions dried in a speed-vac and then the pellets were resuspended in $100 \mu \mathrm{L}$ water. Fifteen microliters were used for the high-performance liquid chromatography analysis.

The GLs studied have been previously described in Arabidopsis. Highperformance liquid chromatography peaks were identified from absorbance at $229 \mathrm{~nm}$ with a UV detector and retention time, in comparison to known standards. Results are presented as $\mu \mathrm{mol} \mathrm{g}^{-1}$ fresh weight based on pure desulfoglucosinolate standards at $229 \mathrm{~nm}$. Each treatment was analyzed in triplicate. Compounds were analyzed on a C-18 column using a 6 min gradient from 1.5 to $5.0 \%(\mathrm{v} / \mathrm{v}$ ) acetonitrile (AN), a 2 min gradient using $5 \%-7 \%$ $\mathrm{AN}$, followed by a 7 min gradient from $7 \%-25 \% \mathrm{AN}$ and a 2 -min gradient transitioning from $25 \%-92 \% \mathrm{AN}$, then $6 \mathrm{~min}$ at $92 \% \mathrm{AN}$, then $1 \mathrm{~min}$ from $92 \%-1.5 \%$ AN and lastly 5 min at $1.5 \%$ AN.

\section{RESULTS}

Stem growth responses during deetiolation in red Russian kale seedlings depend on light wavelengths

Red Russian kale seedling development was first assessed under different light conditions and a variety of fluence rates. Seedlings were allowed to germinate in darkness and then were moved to various wavelengths and three fluence rates, at 24-h intervals (light/ dark; 0 D/96 L, 24 D/72 L, 48 D/48 L and 72 D/24 L). Figure 1 shows the typical seedling response to light with expanded cotyledons and greening and also the repression of hypocotyl elongation. The effect of light on hypocotyl elongation was quantified in Figure 2a$2 d$. White, blue and red light all have comparable effects on the kale responses over the duration of the experiment, leading to stronger stem growth inhibition under higher fluence rates. Far-red light, however, exerts a different effect and promotes the most robust repression, almost independently of the fluence rate, of stem growth rate (Figure $2 \mathrm{a}-2 \mathrm{~d}$ ), with seedlings approximately $10 \%$ the length of dark-grown controls, even at $1 \mu \mathrm{mol} \mathrm{m}{ }^{-2} \mathrm{~s}^{-1}$ after $0 \mathrm{D} / 96 \mathrm{~L}$ treatment (Figure 2a).

Comparison of the differences of hypocotyl length between Figure $2 \mathrm{c}$ and $\mathrm{d}$ suggested the existence of a developmental switch during this time period, which prompted investigation of the 

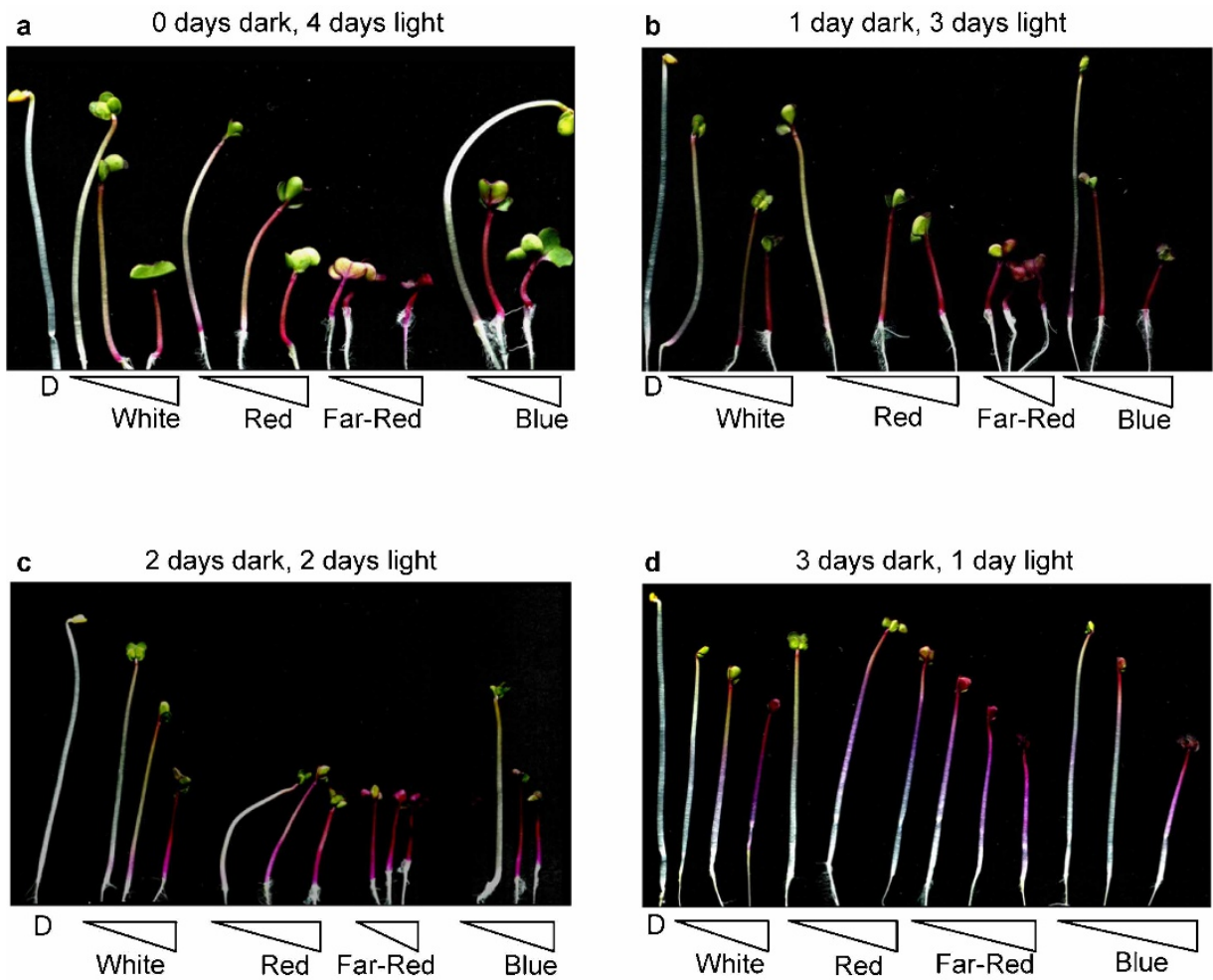

Figure 1. Phenotypes of 4-day-old Red Russian kale seedlings under selective light conditions. (a) Representative seedlings grown for 4 days under dark (D), white $\left(1,10\right.$ and $\left.100 \mu \mathrm{mol} \mathrm{m} \mathrm{m}^{-2} \mathrm{~s}^{-1}\right)$, red $\left(1,25\right.$ and $\left.100 \mu \mathrm{mol} \mathrm{m} \mathrm{m}^{-2} \mathrm{~s}^{-1}\right)$, far-red $\left(1,10\right.$ and $\left.100 \mu \mathrm{mol} \mathrm{m} \mathrm{m}^{-2} \mathrm{~s}^{-1}\right)$ or blue light $(1,20$ and $\left.100 \mu \mathrm{mol} \mathrm{m} \mathrm{m}^{-2} \mathrm{~s}^{-1}\right) ;(\mathbf{b}) 1$ day of darkness and 3 days of light, (c) 2 days of darkness and 2 days of light; (d) 3 days of darkness and 1 day of light.

response to light every $24 \mathrm{~h}$ during 4 days of exposure to an intermediate $\left(10 \mu \mathrm{mol} \mathrm{m} \mathrm{m}^{-2} \mathrm{~s}^{-1}\right)$ fluence rate. As seen in Figure $2 \mathrm{e}$, seedling growth rate under white, red and blue light is relatively slow over the first $48 \mathrm{~h}$ and begins to increase after this time point. Here again an exception is made for far-red, which inhibits growth rate after 2 days of exposure to continuous light (Figure 2e).

Accumulation of anthocyanins and chlorophyll is regulated by the light wavelength and fluence rate

The combination of different treatments of altered darkness/light periods and/or different light wavelengths and fluence rates not only represses stem elongation to different extents but also promotes additional different phenotypic behaviors (Figure 1). The typical seedling response to light is seen in all four conditions tested, but appears to be stronger under $96 \mathrm{~h}$ of light (Figure 1a), with seedlings exhibiting the most cotyledon expansion, and all appearing greener compared to other time points. In addition, generally, higher fluence rates promote darker colors, independently of the wavelength, whereas lower fluence rates result in greener seedlings. These observations suggested that kale seedlings were accumulating different pigments, namely, anthocyanins and chlorophyll, hallmarks of photomorphogenic development. Having in mind consumer-desired colors and potential nutraceutical content, it is important to understand the parameters of pigmentation upon specific light conditions. Therefore, seedlings were grown as described in Figure 1 and anthocyanins and chlorophyll were extracted and measured. The quantitative results from three independent trials of 18 seedlings are shown in Figures 3 and 4. In all cases, the pigments increased with fluence rate, as expected. Anthocyanins accumulated to similar levels under white, red and blue light, but reached much higher levels under the exposure of any fluence rate of far-red light (Figure 3). Chlorophyll accumulated to approximately the same levels in each seedling set (Figure 4). The exception was that accumulation in red light was higher at $25 \mu \mathrm{mol} \mathrm{m} \mathrm{m}^{-2} \mathrm{~s}^{-1}$ than at an intermediate rate in any other light quality (Figure 4).

Balance of red and far-red light on the deetiolation of Red Russian kale seedlings

The modulation of plant growth and development by light is dictated by phytochromes, plant pigments that are generally activated by red light and inactivated by far-red light (reviewed in Chen and Chory, 2011). Under the presence of both wavelengths, a dynamic equilibrium is established, allowing plants to rapidly optimize their response to that particular environment. Figures $2-4$ revealed that far-red and red light exert almost antagonistic effects on photomorphogenic development. Seedlings under far-red light exhibit a stronger suppression of hypocotyl elongation, accompanied by greater accumulation of anthocyanins, as opposed to longer and greener hypocotyls under red light. To further explore the interaction between these two wavelengths on kale growth, seedlings were grown for 4 days under different combinations of simultaneously applied red and far-red light (Figure 5). Consistent with what was observed in Figure $2 \mathrm{a}-2 \mathrm{~d}$, irradiance with a relatively small amount of far-red light, is sufficient to repress hypocotyl elongation (Figure 5a). In addition, the accumulation of anthocyanins is also dramatically higher with decreasing levels of the red to far-light ratio (Figure $5 b$ ). Conspicuous effects are seen in Figure $5 d$, with seedlings showing substantial purple pigment accumulation with less green at lower-red/far-red ratios. The presence of far-red light did not revert the induction of chlorophyll accumulation (Figure 5c) except when far-red light alone was 
a

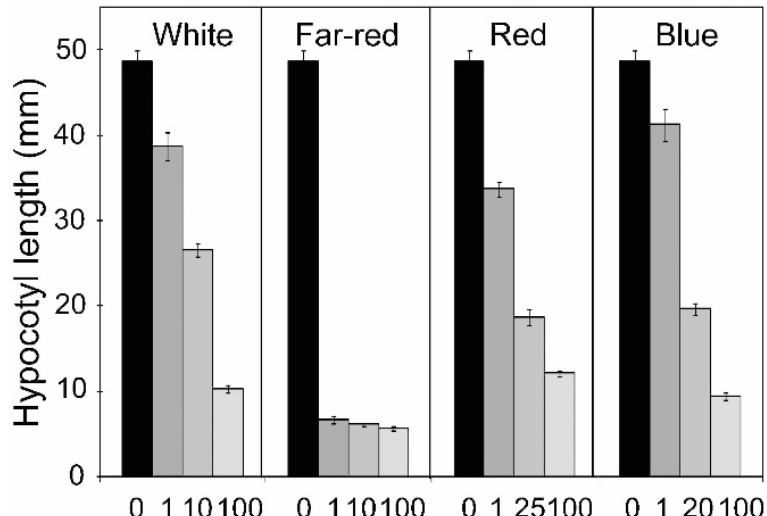

Fluence rate $\left(\mu \mathrm{mol} \mathrm{m} \mathrm{m}^{-2} \mathrm{~s}^{-1}\right)$

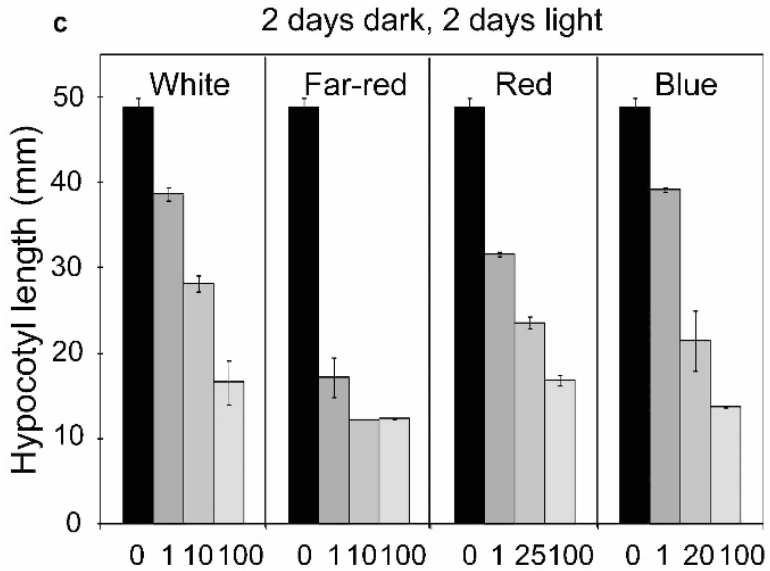

Fluence rate $\left(\mu \mathrm{mol} \mathrm{m} \mathrm{m}^{-2} \mathrm{~s}^{-1}\right)$ b

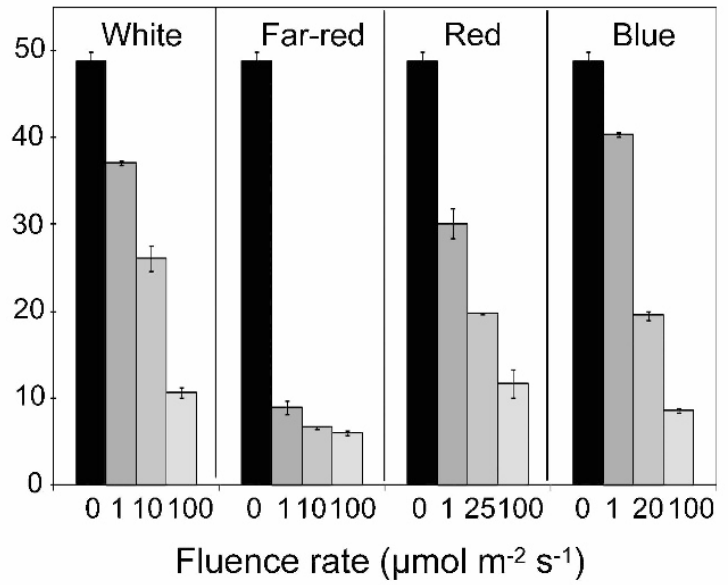

d

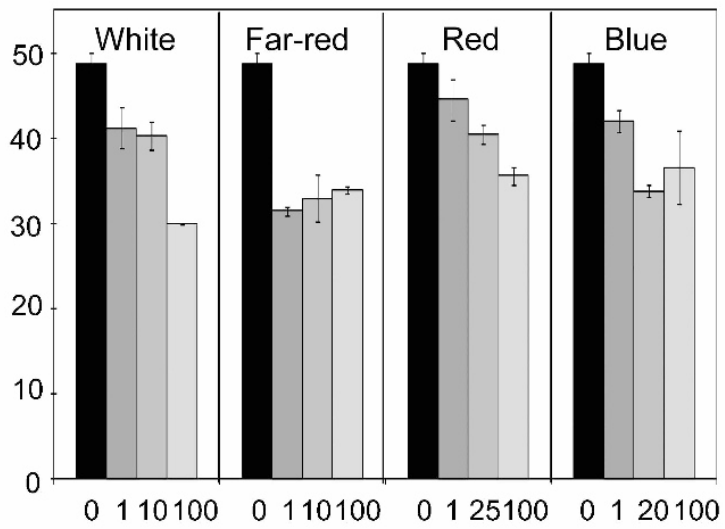

Fluence rate $\left(\mu \mathrm{mol} \mathrm{m} \mathrm{m}^{-2} \mathrm{~s}^{-1}\right)$

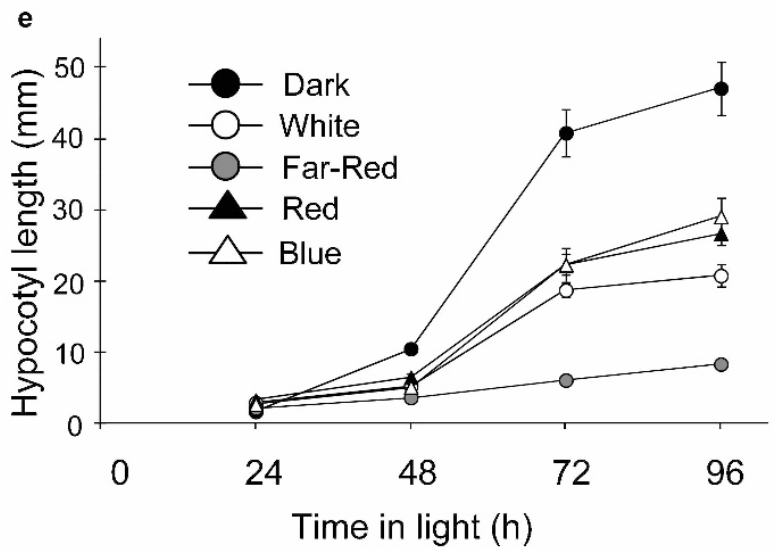

Figure 2. Hypocotyl elongation of Red Russian kale seedlings under selective light conditions. (a) Hypocotyl length after 4 days of exposure to white, far-red, red or blue light with the indicated fluence rates; (b) after 1 day of darkness and 3 d of light; (c) 2 days of darkness and 2 days of light; (d) 3 days of darkness and 1 day of light. (e) Hypocotyl elongation during 4 days of exposure to 0 (closed circles) or $10 \mu \mathrm{mol} \mathrm{m}^{-2} \mathrm{~s}^{-1}$ of white (opened circles), far-red (grey circles), red (closed triangles) or blue (opened triangles) light. Results are representative of three independent experiments. Means \pm s.e., $n=18$. s.e., standard error. 

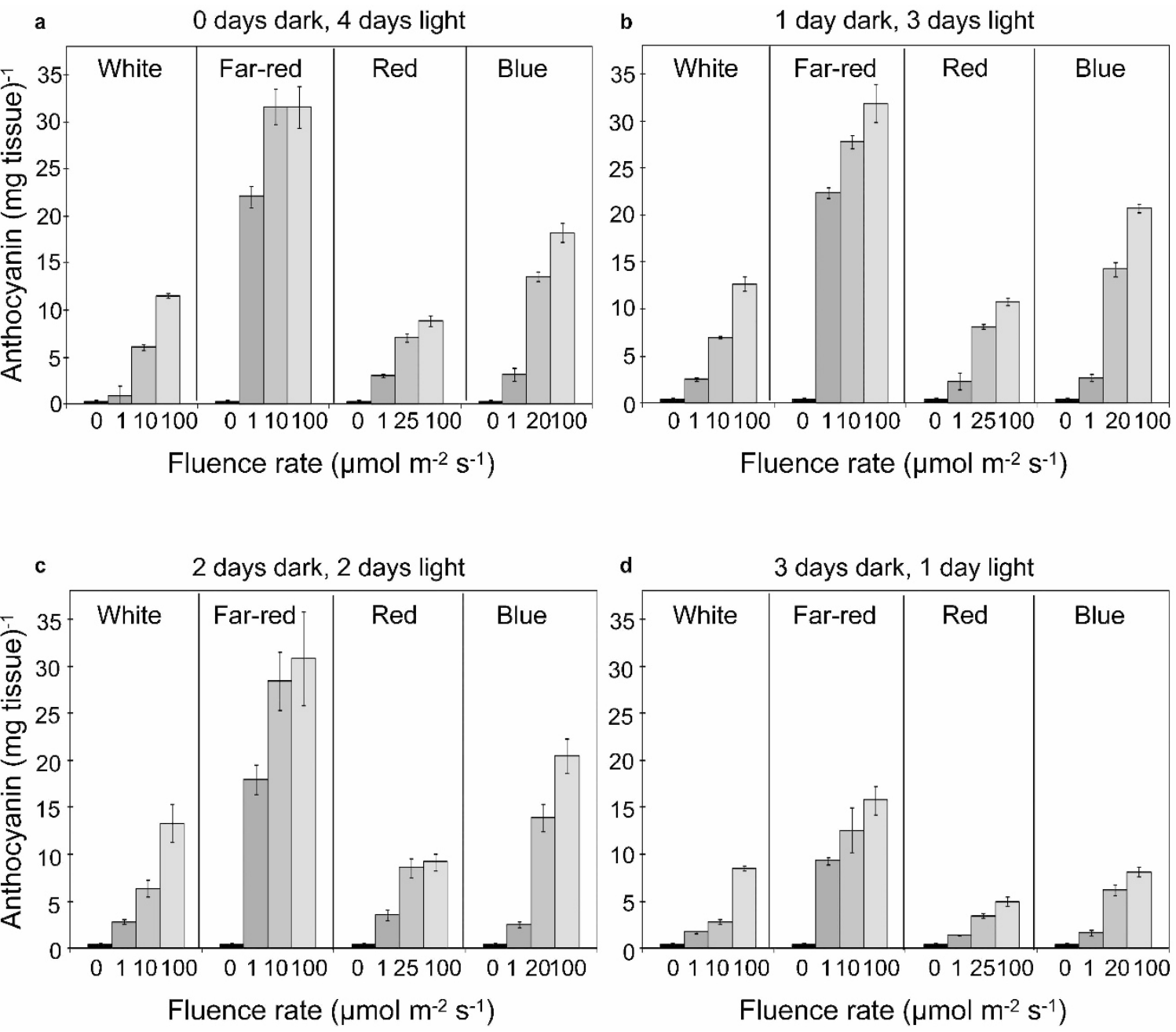

Figure 3. Anthocyanin content in 4-day-old Red Russian kale seedlings under selective light conditions. (a) Anthocyanin levels after 4 days of exposure to white, far-red, red or blue light with the indicated fluence rates; (b) 1 day of darkness and 3 days of light; (c) 2 days of darkness and 2 days of light; (d) 3 days of darkness and 1 day of light. Results represent the average of three independent experiments. Means \pm s.e. s.e., standard error.

applied. Here chlorophyll levels decreased to almost half their levels when red light was present (Figure $5 c$ ).

Addition of blue light to a red/far-red background alters pigment accumulation

Blue light typically induces anthocyanin accumulation. To test the effect of blue supplementation on mixtures of red and far-red light, seedlings were grown as in Figure 5, except that blue light was added. Consistent with the effect of combinations of red and farred light on hypocotyl elongation (Figure $5 \mathrm{a}$ ), the presence of blue light did not augment the strong hypocotyl growth inhibition under high fluence rates (data not shown). The addition of blue light along with red and/or far-red light promoted increased levels of anthocyanins (Figure 6a). Increasing fluence rates of blue light on a red background led to higher levels of anthocyanins. When blue light was applied together with far-red light anthocyanin accumulation was $30 \%$ higher than when far-red light was provided alone (Figures $6 a$ and $3 \mathrm{~b}$ ). The presence of high fluence rates of blue light under a red background was sufficient to induce a $50 \%$ increase in chlorophyll content when compared to the seedlings grown under red light $\left(25 \mu \mathrm{mol} \mathrm{m} \mathrm{m}^{-2} \mathrm{~s}^{-1}\right)$ alone, promoting the highest levels of chlorophyll accumulation measured in this study (Figures $4 \mathrm{~b}$ and $6 \mathrm{~b}$ ).
The total antioxidant capacity and the GL accumulation in kale is affected by the light wavelength

Wavelength-specific effects on anthocyanins and chlorophyll are conspicuous and quantifiable. These data suggest that there may be concomitant changes in antioxidant agents and/or GLs, two classes of compounds with reported health benefits. ${ }^{19-21}$ To test this hypothesis, total antioxidant capacity of kale seedlings grown under different light wavelengths was assessed using the ORAC-FL assay. ${ }^{17,18}$ This method is based on the capacity of any putative antioxidant agent to quench the activity of a peroxyl radical generator that induces a decay in fluorescence emitted by a fluorescent compound. The calculations are made using as a standard the Trolox reagent, a vitamin $\mathrm{C}$ analog, and therefore, the quantitative results indicate the antioxidant capacity of any sample by giving its concentration of Trolox equivalents (TEs). For this test, kale seedlings were grown for 4 days in darkness or under a single fluence rate $\left(50 \mu \mathrm{mol} \mathrm{m} \mathrm{m}^{-2} \mathrm{~s}^{-1}\right)$ of different light wavelengths. The results show that far-red induces the highest TE accumulation (Figure 7), approximately $25 \%$ higher than under white or blue light. In contrast, red light-grown seedlings have a TE concentration much lower than in any other light regime, albeit double the basal level of dark-grown seedlings. 

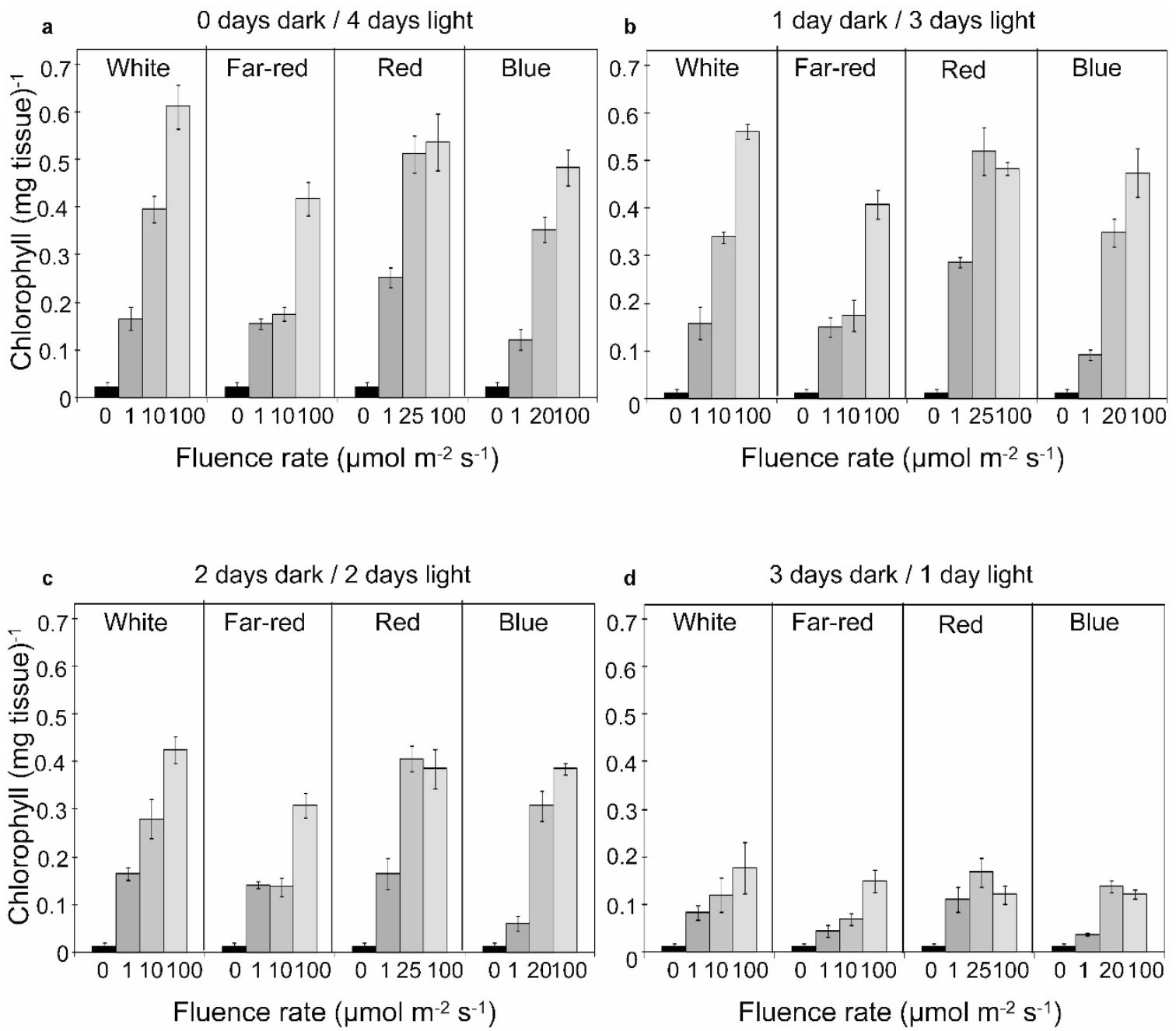

Figure 4. Chlorophyll levels in 4-day-old Red Russian kale seedlings under selective light conditions. (a) Chlorophyll content after 4 days of exposure to white, far-red, red or blue light with the indicated fluence rates; (b) 1 day of darkness and 3 days of light; (c) 2 days of darkness and 2 days of light; (d) 3 days of darkness and 1 day of light. Results represent the average of three independent experiments. Means \pm s.e. s.e., standard error.

GL composition in light-grown kale seedlings was determined by high-performance liquid chromatography. Light treatments were the same as those used for the ORAC-FL tests. GLs may be classified based on their side chain structure into groups. ${ }^{7,21}$ In these trials, two groups were identified-the aliphatic and the indole-and we found differences in the accumulation of total and specific GLs (Table 1). Furthermore, within each group, every specific GL was found to be present in kale grown under all treatments tested.

Treatment with far-red light increased the total aliphatic GL levels by $25 \%$ over dark controls, whereas white light, red and blue light did not induce any significant differences compared to darkness. When looking into specific aliphatic $\mathrm{GL}$, light treatments generally decrease the levels of 4-hydroxy-indolyl-3-methyl-GL (40HI3M), but increase 'unknown' GL species (which could not be precisely resolved) to approximately $100 \%-200 \%$ under white light, red light or blue light conditions compared to dark controls. The only aliphatic species that was significantly induced by a single light treatment was 4-methylsulfinylbutyl-GL (4MSOB), which showed similar levels in all conditions except under far-red light, where a $50 \%$ increase was observed when compared to the dark level. Only blue light, in contrast, significantly affected 4-methylthiobutyl-GL (4MTB) when compared to the other light conditions, resulting in levels $25 \%$ lower than under darkness.
Total indole-based GLs are generally at their highest levels in etiolated seedlings (Table 1) and about the double of what is present with any narrow-bandwidth treatment. Far-red, red and blue light all generate similar total indole GL levels, whereas white light lowers this amount about $40 \%$. The substantial effect of white light on decreasing indole GLs is also visible when looking at specific species, as seen by the $67 \%, 50 \%$ and $85 \%$ decrease in indole-3ylmethyl-GL (I3M), 4-methoxyindol-3-ylmethyl-GL (4MTI3M) and 1-methoxyindol-3-ylmethyl-GL (1MTI3M), respectively, when compared to dark-grown kale. This effect was observed to a similar extent under the rest of the light conditions except for I3M, where far-red, red and blue light did actually decrease it to lower levels than in darkness, but still reached a level $50 \%$ higher than what was measured under white light.

Finally, when total GL levels are examined across all light treatments, the accumulation is the highest in far-red light, with approximately $15 \%-42 \%$ accumulation compared to other light treatments or darkness.

A sequential program to control end-point products We have shown above that controlling light conditions affects different physiological parameters in Red Russian kale. In fact, by 
a

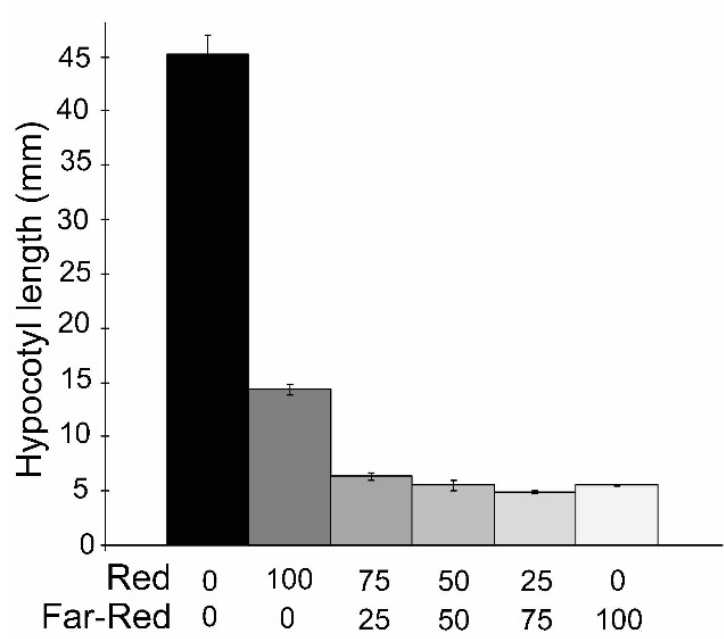

Fluence rate $\left(\mu \mathrm{mol} \mathrm{m} \mathrm{m}^{-2} \mathrm{~s}^{-1}\right)$

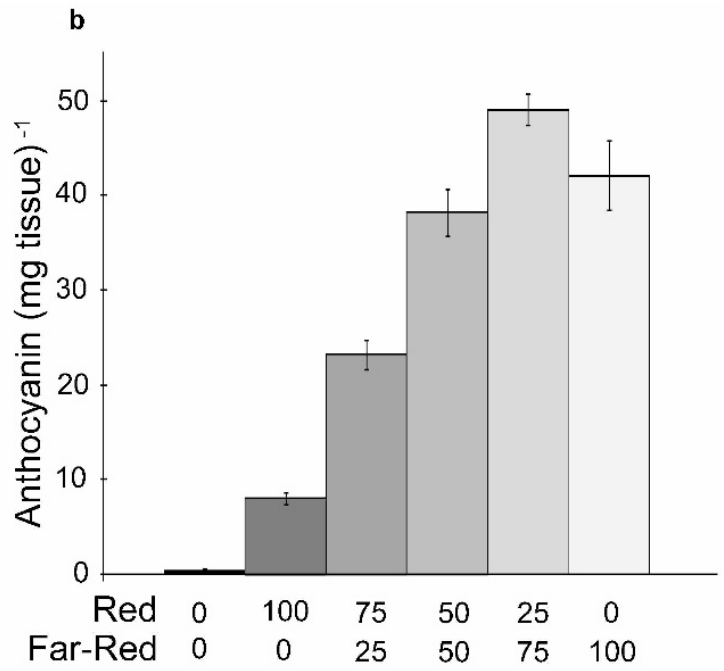

Fluence rate ( $\mu \mathrm{mol} \mathrm{m} \mathrm{m}^{-2} \mathrm{~s}^{-1}$ )
C

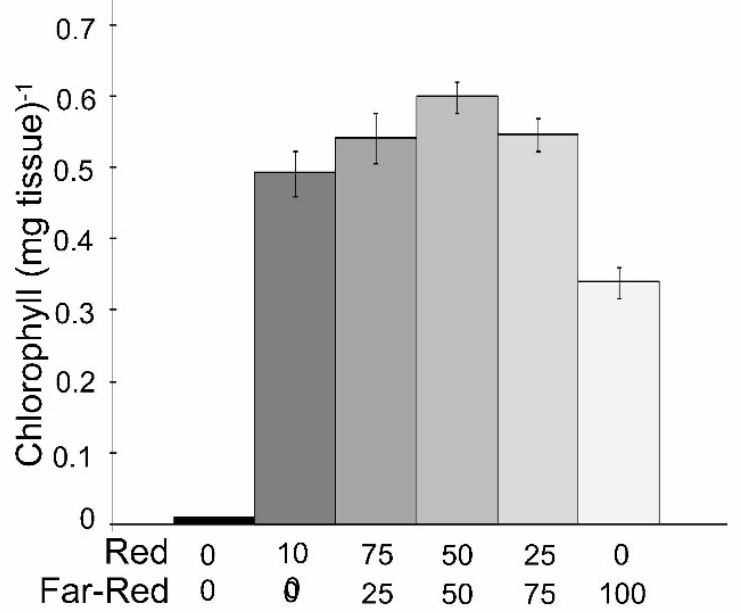

d

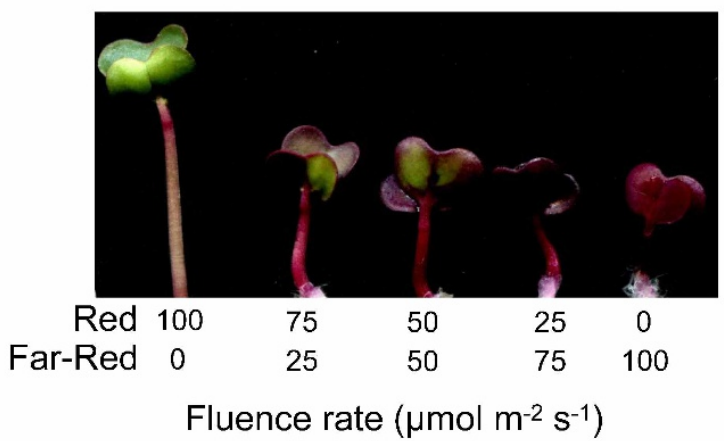

Fluence rate $\left(\mu \mathrm{mol} \mathrm{m} \mathrm{m}^{-2} \mathrm{~s}^{-1}\right)$

Figure 5. Effect of simultaneous red and far-red light irradiance on the growth of Red Russian kale seedlings. (a) Hypocotyl length (representative of three independent experiments; means \pm s.e., $n=18$ ); (b) anthocyanin content (average of three independent experiments, means \pm s.e.); (c) chlorophyll levels (average of three independent experiments, means \pm s.e.); (d) a representative picture of 4-day-old seedlings grown in darkness for 1 day and then 3 days in the indicated red and far-red light fluence rates. s.e., standard error.

changing light wavelengths and fluence rates, we have been able to modulate stem elongation, pigment accumulation and nutrient density. The final test was to integrate the information gained to explore kale sprout plasticity during growth, to reach an 'ideal' kale sprout for market. Four different combinations of light were tested over a period of 4 days. The sequence of treatments (Figure 8a) was designed to promote specific outcomes in the final product. The first treatment (T1) is a control, and includes 3 days of white light treatment following a 24-h dark period to promote stem elongation. Treatment 2 (T2) serves as a transition from T1 to Treatment 3 (T3) and 4 (T4) and uses red instead of white light, at an intermediate fluence rate $\left(25 \mu \mathrm{mol} \mathrm{m} \mathrm{m}^{-2} \mathrm{~s}^{-1}\right)$ that does not repress stem elongation to a strong extent (Figure $2 a-3 c$ ), but is still able to promote chlorophyll accumulation (Figure 4). In T3, the redlight treatment lasts 1 day and the other 2 days are used for a combination of simultaneous far-red and blue light, which promote darker purple seedling colors (Figure 1) and anthocyanin accumulation (Figure 6a) to a stronger level than other light treatments, and also increase the antioxidant capacity of kale (Figure 7). Given the fact that far-red light has a stronger effect on the accumulation of GLs (Table 1), the blue light was switched off in the last day of growth in T4 when compared to T3.

Both white (T1) and red (T2) light treatments repress hypocotyl elongation (Figure $8 \mathrm{~b}$ and $8 \mathrm{c}$ ) as described above (Figure 2b). Exposure to blue and far-red light in T3 and T4 treatments results in shorter seedlings. All four sequential treatments lead, in contrast, to the accumulation of similar chlorophyll levels (Figure 8e). The equal chlorophyll pigmentation is consistent with the similar leaf green coloration seen in all four treatments (Figure 8b), although leaves from T3 and T4 seedlings also show a higher content of 

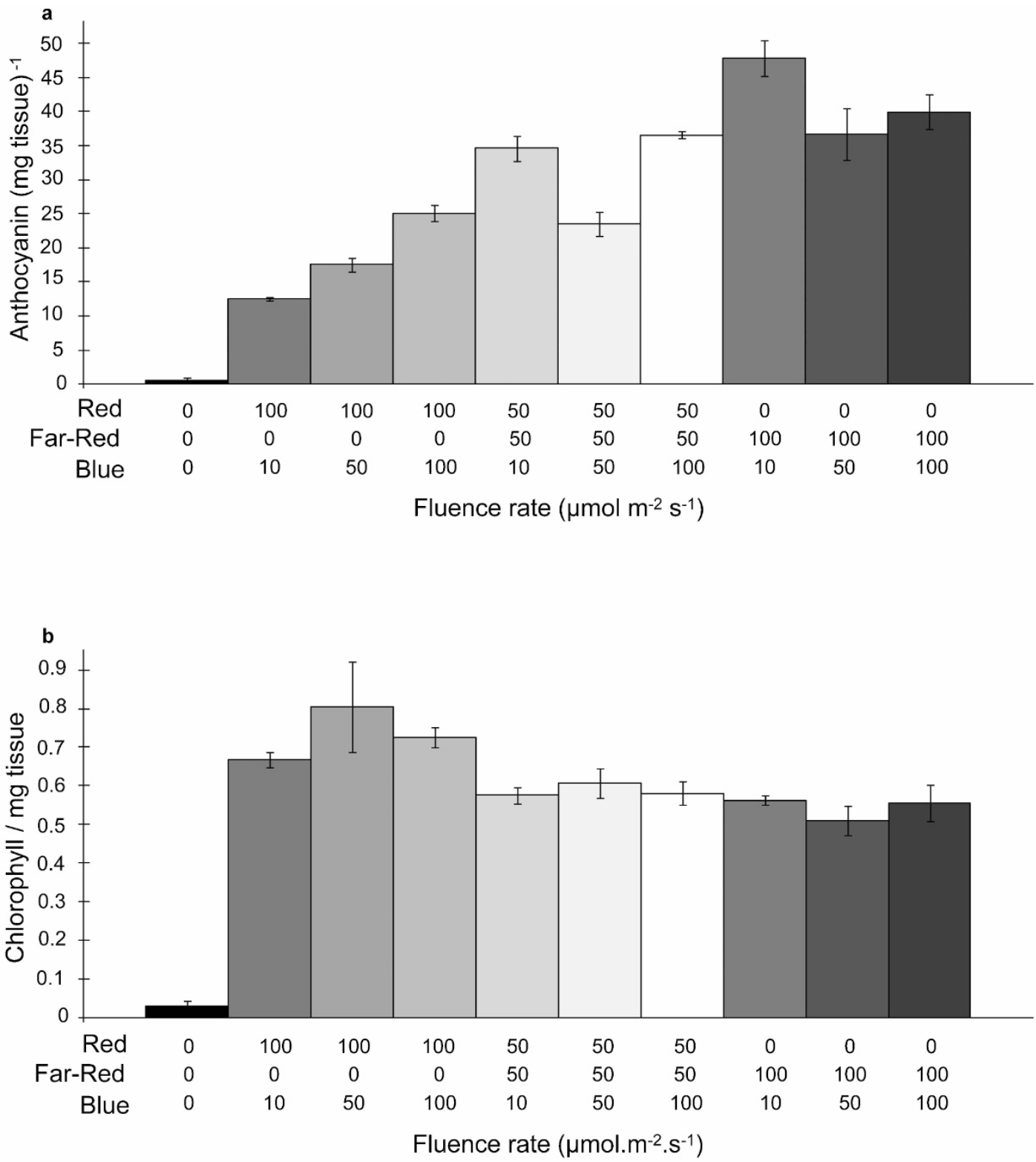

Figure 6. Effect of simultaneous red, far-red and blue light irradiance on the growth of Red Russian kale seedlings. (a) Anthocyanin and (b) chlorophyll levels of 4-day-old seedlings grown in darkness for 1 day and then 3 days under to the indicated red, far-red and blue light conditions. Results represent the average of three independent experiments. Means \pm s.e. s.e., standard error.

purple pigments. The hypocotyls themselves also develop a dark purple color, in contrast to the mild and light purple seen in $\mathrm{T} 1$ and T2, respectively. Consistently, anthocyanin levels in T3 and T4 (Figure $8 \mathrm{~d}$ ) are increased, and $50 \%$ higher than in $\mathrm{T} 1$ and just slightly lower than the levels obtained with a 1-day darkness/3 days of constant far-red or simultaneous far-red and blue wavelengths (Figures $3 \mathrm{~b}$ and 6a).

T1 and T3 treatments result in seedlings with similar antioxidant capacities (Figure $8 \mathrm{f}$ ), with more than $100 \%$ of the TE measured in $\mathrm{T} 2$, but significantly lower than the nearly $100 \mu \mathrm{mol} \mathrm{TE} \mathrm{g}^{-1}$ registered in T4. Both aliphatic and indole-specific and total GL concentrations show a peak under the T3 treatment but decrease in T4.

When comparing GL levels under white light (Table 1) and T1 (Table 2), where the same white light fluence rate was used during the same period of time, we observed that performing the sequential treatments in magenta boxes resulted in higher GL concentrations in opposite to the vertical agar plates. All GL concentrations are induced in T3, as seen by the $45 \%$ increase of aliphatic species from $\mathrm{T} 1$ to $\mathrm{T} 3,83 \%$ in indole GLs and $45 \%$ of the total GLs.

\section{DISCUSSION}

As the cost of solid state, narrow-bandwidth lighting decreases, there is opportunity to use these devices to produce predictable outcomes that improve the value and/or quality of plant products. Many reports have examined the use of LED light to grow products with different qualities. In this study, we demonstrate effects of different treatments on specific aspects of plant growth and development, and then use sequential treatments to produce variation in the final products, in this case using kale sprouts. In preliminary experiments, Red Russian kale sprouts showed great 


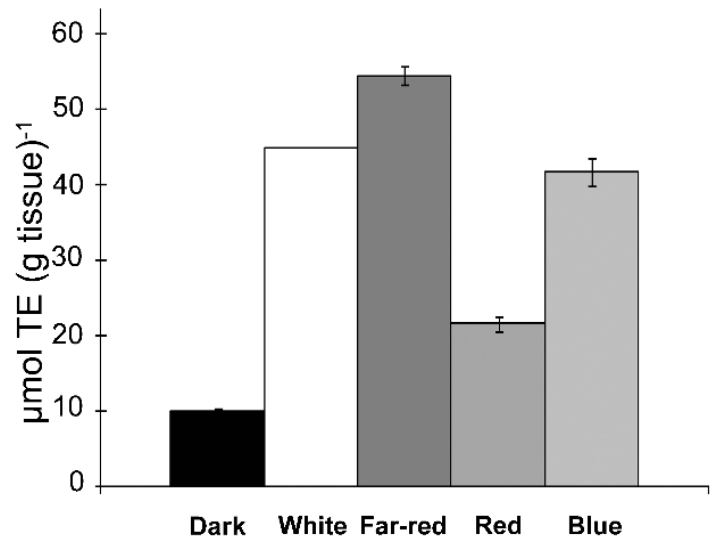

Figure 7. Effect of light wavelength on the antioxidant capacity of Red Russian kale seedlings. TEs in 4-day-old seedlings grown under continuous darkness or $50 \mu \mathrm{mol} \mathrm{m} \mathrm{m}^{-1}$ fluence rate of the indicated light conditions. Results are representative of three independent experiments. Means \pm s.e., $n=3$. s.e., standard error.

phenotypic plasticity in response to the light environment, indicating that it is a suitable model for how light may be used to specifically adjust plant product quality. Manipulation of time in darkness, light wavelengths and fluence rates induced changes in several features, such as stem length, pigment accumulation, antioxidant capacity and GL content.

The variation in growth, development and metabolism in response to external cues has been documented, but is not often considered in the actual production of horticultural crops. Lee et al. ${ }^{10}$ induced significant increases in key phytochemicals with heat-shock treatments. Different combinations of altered $\mathrm{CO}_{2}$ salinity and light levels influence lettuce antoxidant activity. ${ }^{22}$ Salinity stress has also been shown to influence different metabolites in radish sprouts. ${ }^{23}$ The goal of this work was to demonstrate how light could be used to manipulate high-value product traits.

In Arabidopsis thaliana, identification of light sensing mutants and their preliminary characterization by Koornneef et al. ${ }^{24}$ eventually led to the discovery of photoreceptor-encoding genes. Over the next decades, light control of Arabidopsis growth and development led to extensive characterization of how to control stem growth, expansion of leaves, development of pigmentation, control of gene expression and accumulation of metabolites. While the mechanisms are understood, they are now just being translated to crops of economic interest. The expansion of Arabidopsis principles to other Brassica sprouts is particularly of interest as light can affect consumer-desired attributes and at the same time, provide information about the similarities and differences between the model and the crop.

Light studies in mature plants have demonstrated that specific light treatments can influence accumulation of carotenoids, ${ }^{13,25-27}$ anthocyanins ${ }^{28,29}$ and chlorophyll. ${ }^{30}$ The first tests performed here examined how conspicuous seedling traits such as hypocotyl growth rate, chlorophyll accumulation, anthocyanin levels and cotyledon expansion are affected by time of growth in darkness followed by transfer to various fluence rates of light. Generally, typical photomorphogenic responses are observed with respect to inhibition of stem elongation, cotyledon expansion and accumulation of pigments, in end-point analyses. Such findings are consistent with what has been observed in $A$. thaliana seedlings. However, this kale seedling shows some important differences. Observation in Figure 1 and quantitative results in Figure 2 show that most of the elongation growth is occurring after 2 days in darkness. In contrast, Arabidopsis grows rapidly in darkness shortly after germination and elongation rates slow thereafter. ${ }^{31}$ Red Russian kale elongates slowly at first and then more rapidly with establishment (Figure 2e). The effects of white, red and blue light are generally comparable when comparing seedling morphology, with a few exceptions. White light has limited effects on hypocotyl elongation at low fluence rates. This finding is important because application of narrow-bandwidth light can potentially use lower fluence rates and less energy to obtain a stronger effect. Like Arabidopsis, far-red light has a strong effect on stem growth rate inhibition, yet the effect is even observed here at the lowest fluence rates. This finding suggests a hypersensitivity to far-red light.

Anthocyanins have been associated with healthful benefits and provide an attractive coloration to fruit and vegetable products. Black raspberry promoter demethylation-mediated cancer protective effects have been shown to be in part based on anthocyanin bioactivities. ${ }^{32}$ In the mulberry plant and in sweet potato, anthocyanins are known to act as hypoglycemic agents, suggesting their use in diabetes prevention. ${ }^{33,34}$ Demonstrated anti-inflammatory and antimicrobial activities of black soybean anthocyanins suggest these pigments as good candidates for a synergistic usage with administered antibiotics. ${ }^{35}$ The natural availability of anthocyanin metabolic enzymes in different human tissues further potentiates the use of anthocyanin-rich products in target site therapies. ${ }^{36}$

Seedling pigmentation can be affected by various light qualities and quantities, with higher fluence rates of blue and UV light exerting the strongest effect. ${ }^{14,29,37}$ In the examination of Red Russian

Table 1. Steady-state accumulation of GLs in specific light conditions. Data are presented as the mean of three independent biological replicates with standard error of the mean. Letter notations indicate significantly different values (one-way ANOVA, $p<0.05$ )

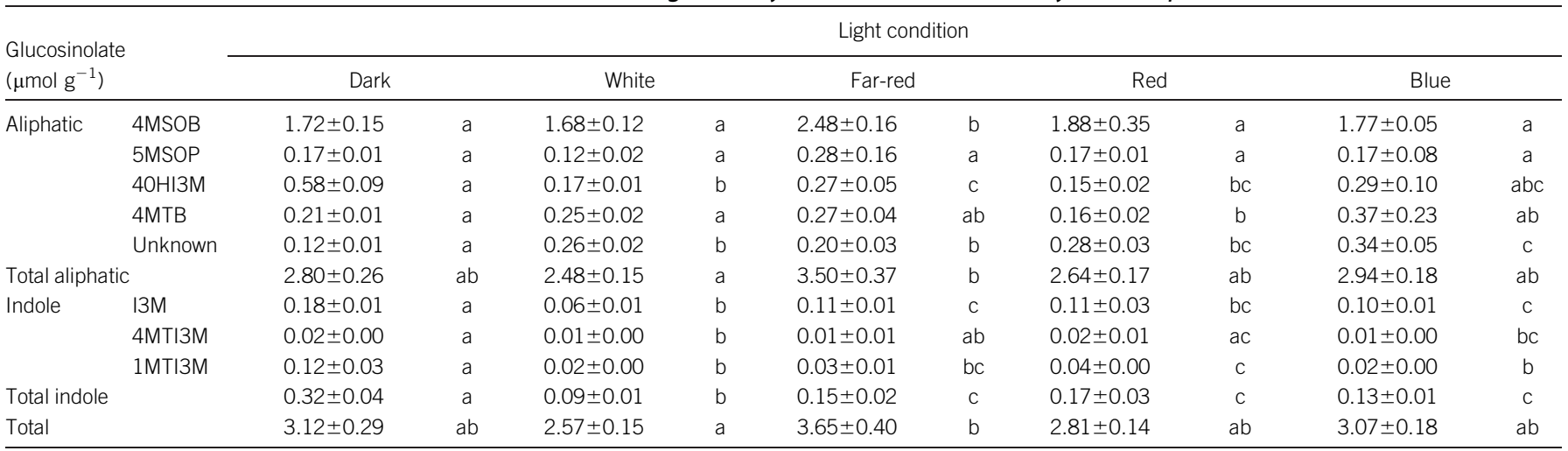

Abbreviations: 4MSOB, 4-methylsulfinylbutyl-GL; 5MSOP, 5-methylsulfinylpentyl-GL; 4OHI3M, 4-hydroxy-indolyl-3-methyl-GL; 4MTB, 4-methylthiobutyl-GL, I3M, indole3-ylmethyl-GL; 4MTI3M, 4-methoxyindol-3-ylmethyl-GL; 1MTI3M, 1-methoxyindol-3-ylmethyl-GL. 


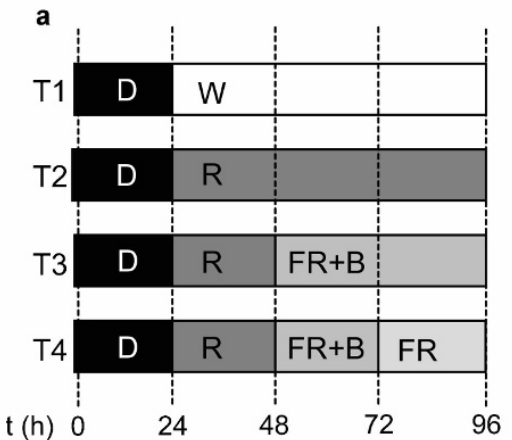

b
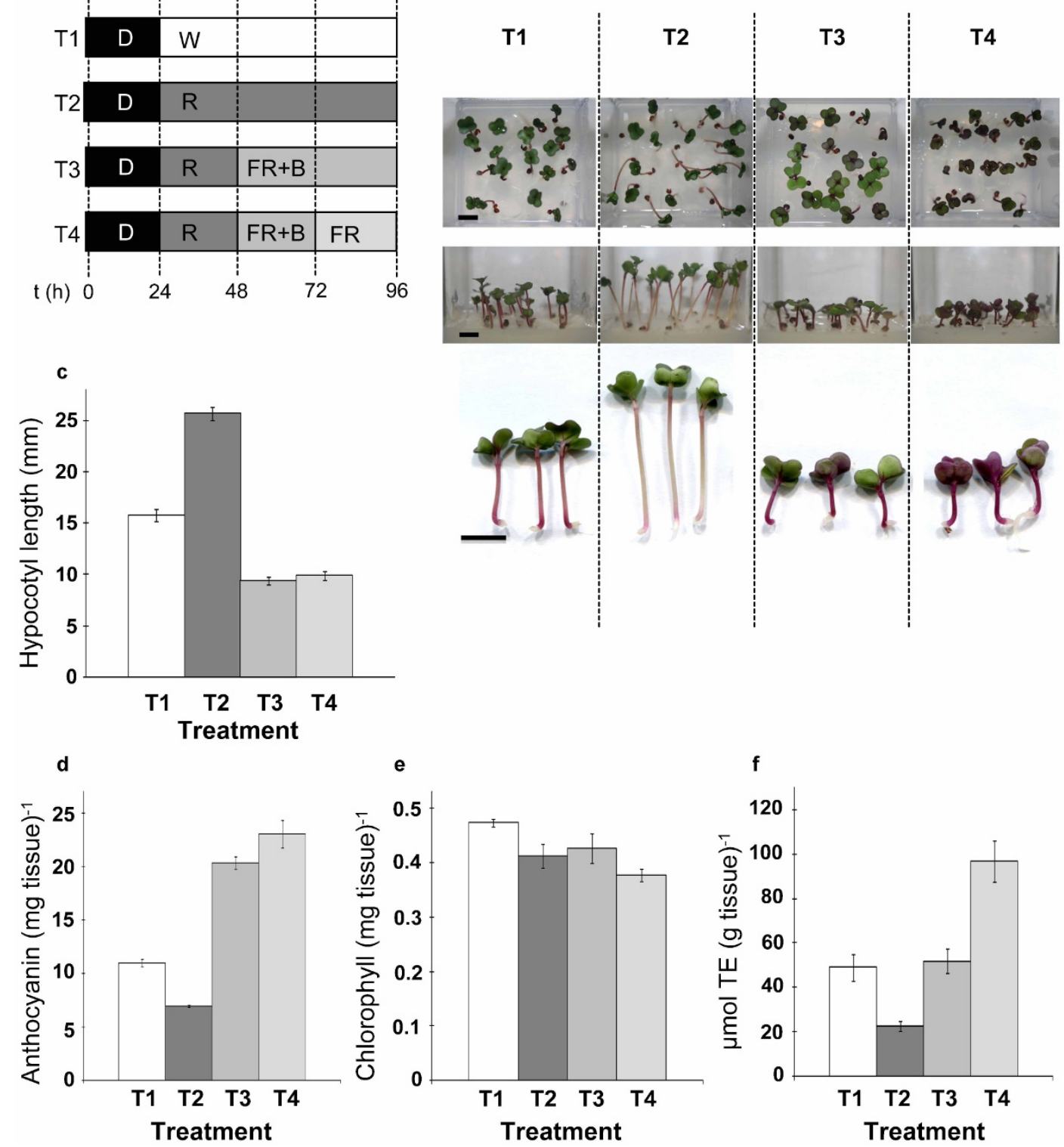

Figure 8. Growth of Red Russian kale under optimized sequential light treatments. (a) Scheme representing the four treatments (T1 to T4) of light used on seedlings grown for 4 days. Transitions every $24 \mathrm{~h}$ are indicated and a change in color represents a change in a light condition. $\mathrm{D}$, dark; W, white light $\left(50 \mu \mathrm{mol} \mathrm{m} \mathrm{m}^{-2} \mathrm{~s}^{-1}\right) ; \mathrm{R}$, red light $\left(25 \mu \mathrm{mol} \mathrm{m} \mathrm{m}^{-2} \mathrm{~s}^{-1}\right) ; \mathrm{FR}$, far-red light $\left(50 \mu \mathrm{mol} \mathrm{m}^{-2} \mathrm{~s}^{-1}\right) ; \mathrm{B}$, blue light $\left(50 \mu \mathrm{mol} \mathrm{m} \mathrm{m}^{-2} \mathrm{~s}^{-1}\right)$. (b) Representative pictures; (c) hypocotyl elongation; (d) anthocyanin accumulation; (e) chlorophyll levels; (f) antioxidant capacity of kale seedlings from all four treatments. Scale bars $=1 \mathrm{~cm}$.

kale seedlings, all wavelengths induced anthocyanin accumulation, even at low fluence rates. Our results (Figure 3 ) also show that while all light conditions induce anthocyanin accumulation, the far-red treatments alone add substantial anthocyanin pigmentation to the seedling. Compared to blue light, the lowest fluence rate tested $\left(1 \mu \mathrm{mol} \mathrm{m} \mathrm{m}^{-2} \mathrm{~s}^{-1}\right)$ was about 10 times more effective at generating anthocyanin accumulation, whereas a $10 \mu \mathrm{mol} \mathrm{m} \mathrm{m}^{-2} \mathrm{~s}^{-1}$ treatment led to a two-fold increase (Figure 3a). Chlorophyll accumulation was comparable in all light conditions, both in respect to seedling developmental competence and in fluence rate response. The exception was that red light caused significantly more accumulation under a lower fluence rates $\left(25 \mu \mathrm{mol} \mathrm{m} \mathrm{m}^{-2} \mathrm{~s}^{-1}\right)$.

The application of narrow-bandwidth light technology in control of plant growth and development lies in application of precise mixes of light through time to optimize the production of desired traits. The concept of 'steady signaling states' implies that a given light condition may bring gene expression and metabolism into a predictable range. ${ }^{38}$ Consistent with this concept, the ratio of red to far-red light was tested, as a steady-state equilibrium of active phytochrome and input through phytochrome A may allow optimization of pigment accumulation. Our results (Figure 5) indicate that far-red and red with a balance of 3:1 at these fluence rates provides a maximum level of anthocyanins and chlorophylls. Chlorophyll does not accumulate well under far-red light alone, owing to the effect of far-red light on chloroplast development. ${ }^{39}$ In combination with blue light (Figure 6), far-red light produces more anthocyanins per $g$ tissue than with far-red light alone (Figure 3), suggesting co-action of multiple sensory systems. Again, far-red light induces the strongest anthocyanin response, while having an inhibitory effect on chlorophyll accumulation. 
Table 2. Steady-state accumulation of GL in sequential light treatments. Data are presented as the mean of three independent biological replicates with standard error of the mean. Letter notations indicate significantly different values (one-way ANOVA, $p<0.05$ )

\begin{tabular}{|c|c|c|c|c|c|c|c|c|c|}
\hline & & & & & Seq & atment & & & \\
\hline Aliphatic & $4 \mathrm{MSOB}$ & $3.42 \pm 0.70$ & a & $3.92 \pm 0.33$ & a & $4.98 \pm 0.38$ & a & $4.49 \pm 0.35$ & a \\
\hline & $40 \mathrm{HI} 3 \mathrm{M}$ & $1.11 \pm 0.27$ & $a b$ & $1.18 \pm 0.11$ & $a b$ & $1.47 \pm 0.09$ & a & $0.73 \pm 0.19$ & b \\
\hline & 4MTB & $0.76 \pm 0.11$ & $\mathrm{ac}$ & $0.98 \pm 0.08$ & $a b$ & $1.16 \pm 0.11$ & b & $0.52 \pm 0.07$ & c \\
\hline & Unknown & $0.05 \pm 0.01$ & a & $0.13 \pm 0.01$ & $\mathrm{~b}$ & $0.08 \pm 0.01$ & a & $0.03 \pm 0.02$ & a \\
\hline & $4 \mathrm{MTI} \mathrm{M}$ & $0.02 \pm 0.00$ & a & $0.02 \pm 0.00$ & $a b$ & $0.03 \pm 0.00$ & $\mathrm{~b}$ & $0.01 \pm 0.01$ & a \\
\hline & $1 \mathrm{MTI} \mathrm{MM}$ & $0.03 \pm 0.01$ & a & $0.07 \pm 0.01$ & $b$ & $0.06 \pm 0.01$ & $\mathrm{~b}$ & $0.02 \pm 0.01$ & a \\
\hline Total ind & & $0.06 \pm 0.01$ & a & $0.10 \pm 0.01$ & b & $0.11 \pm 0.01$ & $\mathrm{~b}$ & $0.03 \pm 0.03$ & a \\
\hline Total & & $5.73 \pm 1.17$ & $a b$ & $6.67 \pm 0.55$ & $a b$ & $8.33 \pm 0.51$ & a & $5.92 \pm 0.35$ & $\mathrm{~b}$ \\
\hline
\end{tabular}

Abbreviations: 4MSOB, 4-methylsulfinylbutyl-GL; 5MSOP, 5-methylsulfinylpentyl-GL; 4OHI3M, 4-hydroxy-indolyl-3-methyl-GL; 4MTB, 4-methylthiobutyl-GL; I3M, indole3-ylmethyl-GL; 4MTI3M, 4-methoxyindol-3-ylmethyl-GL; 1MTI3M, 1-methoxyindol-3-ylmethyl-GL.

The accumulation of specific secondary metabolites can be controlled by light. ${ }^{28}$ General antioxidants are induced by some light conditions. For example, UV-B and UV-C stimulates phenolics, stilbenes and overall antioxidant activity in pigeon pea (Cajanus cajan) leaves. ${ }^{40,41}$ A supplementary UV-B treatment also affects the total antioxidant activity in basil. ${ }^{42}$ Here the ORAC-FL method was employed to estimate general antioxidant capacity in light-treated Brassica seedlings. The results in Fig. 7 show that far-red light also elevates the antioxidant potential of treated seedlings, with white light also producing high levels. The latter result suggests that input through multiple light sensory pathways is required to maximize production, while the former shows that activation of phyA is sufficient to attain the same effect.

Brassicas are known to be particularly enriched in GLs, compounds that contribute to their flavors and have been associated with healthful effects. ${ }^{43}$ GLs have been shown to be affected by light treatments in mature kales ${ }^{13}$ and in radish hypocotyls, ${ }^{44}$ with blue light supplementation leading to higher levels. In this study, light increased the levels of most aliphatic GLs, with the exception of $40 \mathrm{HI} 3 \mathrm{M}$, which is dark abundant (Table 1). Generally, the effects are not statistically significant, with some exceptions such as the increase in $4 \mathrm{MSOB}$ by far-red and in unclassified aliphatic GLs by blue light. Indole-based GLs show their greatest abundance in darkness, and all light treatments drive down their accumulation. The indole GLs are associated with effects on enzymes in the cell cycle and potential anti-cancer effects. ${ }^{45}$ Overall, these results indicate that only minor differences are observed in response to different light treatments, and are likely not physiologically relevant. In addition, it is possible that the GL levels in mature kale plants differ from the numbers measured in young seedlings. Changes in GL relative compositions throughout plant development and in selected tissues have been reported in different Brassica crops $^{46}$ and in Arabidopsis. ${ }^{47}$ Rather than manipulating GL content with light, it may be more favorable for growers to focus on producing attractive seedlings, as increased consumption will introduce higher amounts of GLs than a light treatment can install.

These findings demonstrate that this variety exhibits environmental plasticity that may be exploited to control plant form and content to generate a higher-value product for growers. Therefore, we developed an integrative approach to generate a kale sprout with an acceptable size, a desirable and color and optimized antioxidant levels (Figure 8a). The first treatment matches standard production characteristics-germination in darkness followed by greening and cotyledon development under white light. Another group of seedlings was germinated in darkness to promote stem elongation, and then was transferred to an intermediate fluence rate of red light. Red light promoted chlorophyll accumulation and while still allowing elongation growth, leading to green sprouts with long stems and some accumulation of anthocyanins. The similar chlorophyll levels obtained in the two treatments using blue and far-red light can either reflect the inclusion of a 24-h red-light pulse or the combination of blue and far-red light inductive effects on this pigment accumulation, despite the fact that this red-light pulse was insufficient to prevent stem elongation inhibition to a large extent. The addition of blue light led to a strong production of anthocyanins and total GLs (Table 2) and a final far-red treatment drove the production of antioxidants, but decreased GL concentrations. This result contrasts with the measurements under singlelight wavelengths (Table 1), where the higher antioxidant capacity (Figure 7) correlated with the highest GL levels. The lack of further knowledge on the molecular bases for such responses supports the idea that, at the moment, manipulating GL's levels is not the easiest and best approach for kale growers. However, it is interesting to note that the magnitudes of GL concentrations differ among experimental conditions, as they were considerable lower in seedlings grown in vertical plates under single-light wavelengths as opposed to the culture boxes used in the sequential treatments. Therefore, using different methodologies to grow kale sprouts should be considered when developing final products with unique properties.

The most relevant finding toward application is that this limited set of conditions can produce a variety of plant products from a single genetic background. The concept is exciting because a small grower with a simple light infrastructure can modulate colors, size and nutrition to add value to a single genotype. It allows one set of seeds, management and production practices to be maintained, with an end product of remarkably different seedlings.

Aside from the applied facets of this research, the results suggest some basic dividends that may expand our understanding of light signaling in the process of crop domestication. The results from this work raise the compelling hypothesis that Red Russian kale's far-red hypersensitivity may be the foundation for the selection. Red Russian kale is a variety recognized for its purple-gray leaves and strongly pigmented petioles and leaf veins. This instance may be another case where variation in aspects of light signaling led to selection and eventually domestication. The mechanistic basis for the far-red hypersensitivity is currently being investigated.

In this report, regimens of narrow-bandwidth light were used to control several valued traits of a nutrient dense food. The work applies the basic biology derived from a laboratory plant to a 
specialty crop, translating principles learned to generate attractive novel colors and nutraceuticals to consumers. This study also demonstrates the range of phenotypes that may be extracted from a given genotype, simply by modulating ambient light conditions. The findings pave the way for additional experiments that use narrow-bandwidth lighting or supplementation to affect traits that add value to small format crops.

\section{CONFLICT OF INTEREST}

The authors declare no conflict of interest.

\section{ACKNOWLEDGMENTS}

This work was performed under light sources purchased through the National Science Foundation Grant \# IOS-0746756 (KMF). We thank K Cline and M McCaffery for providing the fluorometer for the ORAC assay, and S Chen, N Zhu and H Wan for support in GL measurement.

\section{REFERENCES}

1 Manchali S, Murthy KN, Patil BS. Crucial facts about health benefits of popular cruciferous vegetables. J Funct Foods 2012; 4: 94-106.

2 Björkman M, Klingen I, Birch AN et al. Phytochemicals of Brassicaceae in plant protection and human health-influences of climate, environment and agronomic practice. Phytochemistry 2011; 72: 538-556.

3 Fahey JW, Zhang Y, Talalay P. Broccoli sprouts: an exceptionally rich source of inducers of enzymes that protect against chemical carcinogens. Proc Natl Acad Sci USA 1997; 94: 10367-10372.

4 Gu Y, Guo Q, Zhang L, Chen Z, Han Y, Gu Z. Physiological and biochemical metabolism of germinating broccoli seeds and sprouts. J Agric Food Chem 2012; 60: 209-213.

5 Abbaoui B, Riedl KM, Ralston RA et al. Inhibition of bladder cancer by broccoli isothiocyanates sulforaphane and erucin: characterization, metabolism, and interconversion. Mol Nutr Food Res 2012; 56: 1675-1687.

6 Brooks JD, Paton VG, Vidanes G. Potent induction of phase 2 enzymes in human prostate cells by sulforaphane. Cancer Epidemiol Biomarkers Prev 2001; 10: 949 954.

7 Dinkova-Kostova AT, Fahey JW, Benedict AL et al. Dietary glucoraphanin-rich broccoli sprout extracts protect against UV radiation-induced skin carcinogenesis in SKH-1 hairless mice. Photochem Photobiol Sci 2010; 9: 597-600.

8 Kensler TW, Ng D, Carmella SG et al. Modulation of the metabolism of airborne pollutants by glucoraphanin-rich and sulforaphane-rich broccoli sprout beverages in Qidong, China. Carcinogenesis 2012; 33: 101-107.

9 Shapiro TA, Fahey JW, Dinkova-Kostova AT et al. Safety, tolerance, and metabolism of broccoli sprout glucosinolates and isothiocyanates: a clinical phase I study. Nutr Cancer 2006; 55: 53-62.

10 Lee MJ, Lim S, Kim J, Oh MM. Heat shock treatments induce the accumulation of phytochemicals in kale sprouts. Korean J Hort Sci Technol 2012; 30: 509-518.

11 Li S, Schonhof I, Krumbein A, Li L, Stützel H, Schreiner M. Glucosinolate concentration in turnip (Brassica rapa ssp. rapifera L.) roots as affected by nitrogen and sulfur supply. J Agric Food Chem 2007; 55: 8452-8457.

12 Mewis I, Schreiner M, Nguyen CN et al. UV-B irradiation changes specifically the secondary metabolite profile in broccoli sprouts: induced signaling overlaps with defense response to biotic stressors. Plant Cell Physiol 2012; 53: 1546-1560.

13 Lefsrud M, Kopsell D, Sams C. Irradiance from distinct wavelength light-emitting diodes affect secondary metabolites in kale. Hortscience 2008; 43: 2243-2244.

14 Kopsell D, Sams C. Increases in shoot tissue pigments, glucosinolates, and mineral elements in sprouting broccoli after exposure to short-duration blue light from light emitting diodes. J Am Soc Hort Sci 2013; 138: 31-37.

15 Neff MM, Chory J. Genetic interactions between phytochrome A, phytochrome B, and cryptochrome 1 during Arabidopsis development. Plant Physiol 1998; 118: 27-35.

16 Moran R, Porath D. Chlorophyll determination in intact tissues using $\mathrm{N}, \mathrm{N}-$ dimethylformamide. Plant Physiol 1980; 65: 478-479.

17 Cao G, Alessio HM, Cutler RG. Oxygen-radical absorbance capacity assay for antioxidants. Free Radic Biol Med 1993; 14: 303-311.

18 Ou B, Hampsch-Woodill M, Prior RL. Development and validation of an improved oxygen radical absorbance capacity assay using fluorescein as the fluorescent probe. J Agric Food Chem 2001; 49: 4619-4626.

19 Gülçin I. Antioxidant activity of food constituents: an overview. Arch Toxicol 2012; 86: 345-391.

20 Traka M, Mithen R. Glucosinolates, isothiocyanates and human health. Phytochem Rev 2009; 8: 269-282.
21 Prakash D, Gupta C. Glucosinolates: the phytochemicals of nutraceutical importance. J Complement Integr Med 2012; 9: Article 13.

22 Pérez-López U, Miranda-Apodaca J, Muñoz-Rueda A, Mena-Petite A. Lettuce production and antioxidant capacity are differentially modified by salt stress and light intensity under ambient and elevated $\mathrm{CO}_{2}$. J Plant Physiol 2013; 170: $1517-$ 1525.

23 Yuan G, Wang X, Guo R, Wang Q. Effect of salt stress on phenolic compounds, glucosinolates, myrosinase and antioxidant activity in radish sprouts. Food Chem 2010; 121: 1014-1019.

24 Koornneef M, Rolff E, Spruit CJ. Genetic control of light-inhibited hypocotyl elongation in Arabidopsis thaliana (L.) Heynh. Z Pflanzenphysiol 1980; 100: 147-160.

25 Becatti E, Petroni K, Giuntini D et al. Solar UV-B radiation influences carotenoid accumulation of tomato fruit through both ethylene-dependent and -independent mechanisms. J Agric Food Chem 2009; 57: 10979-10989.

26 Tuan P, Park N, Park W et al. Carotenoids accumulation and expression of carotenogenesis genes during seedling and leaf development in Chinese cabbage (Brassica rapa subsp pekinensis). Plant Omics 2012; 5: 143-148.

27 Lee WL, Huang JZ, Chen LC, Tsai CC, Chen FC. Developmental and LED light source modulation of carotenogenic gene expression in Oncidium gower ramsey flowers. Plant Mol Biol Rep 2013; 31: 1433-1445.

28 Samuolienè G, Brazaitytè A, Jankauskienè J et al. LED irradiance level affects growth and nutritional quality of Brassica microgreens. Cent Eur J Biol 2013; 8: 1241-1249.

29 Zhou B, Li Y, Xu Z, Yan H, Homma S, Kawabata S. Ultraviolet A-specific induction of anthocyanin biosynthesis in the swollen hypocotyls of turnip (Brassica rapa). J Exp Botany 2007; 58: 1771-1781.

30 Fan X, Zang J, Xu Z et al. Effects of different light quality on growth, chlorophyll concentration and chlorophyll biosynthesis precursors of non-heading Chinese cabbage (Brassica campestris L.). Acta Physiol Plant 2013; 35: 2721-2726.

31 Gendreau E, Traas J, Desnos T, Grandjean O, Caboche M, Hofte H. Cellular basis of hypocotyl growth in Arabidopsis thaliana. Plant Physiol 1997; 114: 295-305.

32 Wang LS, Kuo CT, Cho SJ et al. Black raspberry-derived anthocyanins demethylate tumor suppressor genes through the inhibition of DNMT1 and DNMT3B in colon cancer cells. Nutr Cancer 2013; 65: 118-125.

33 Zhao JG, Yan QQ, Lu LZ, Zhang YQ. In vivo antioxidant, hypoglycemic, and antitumor activities of anthocyanin extracts from purple sweet potato. Nutr Res Pract 2013; 7: 359-365

34 Gao Y, Li J, Strickland E et al. An arabidopsis promoter microarray and its initial usage in the identification of HY5 binding targets in vitro. Plant Mol Biol 2004; 54 683-699.

35 Yoon B, Bae W, Choi $\mathrm{Y}$ et al. The anti-inflammatory and antimicrobial effects of anthocyanin extracted from black soybean on chronic bacterial prostatitis rat model. Chin J Integr Med; e-pub ahead of print October 2013; doi:10.1007/ s11655-013-1547-y.

36 Mallery SR, Budendorf DE, Larsen MP et al. Effects of human oral mucosal tissue, saliva, and oral microflora on intraoral metabolism and bioactivation of black raspberry anthocyanins. Cancer Prev Res 2011; 4: 1209-1221.

37 Wang Y, Zhou B, Sun M, Li Y, Kawabata S. UV-A light induces anthocyanin biosynthesis in a manner distinct from synergistic blue + UV-B light and UV-A/ blue light responses in different parts of the hypocotyls in turnip seedlings. Plant Cell Physiol 2012; 53: 1470-1480.

38 Folta KM, Childers KS. Light as a growth regulator: controlling plant biology with narrow-bandwidth solid-state lighting systems. HortScience 2008; 43: 1957-1964.

39 Barnes SA, Nishizawa NK, Quaggio RB, Whitelam GC, Chua NH. Far-red light blocks greening of Arabidopsis seedlings via a phytochrome A-mediated change in plastid development. Plant Cell 1996; 8: 601-615.

40 Wei ZF, Luo M, Zhao CJ et al. UV-induced changes of active components and antioxidant activity in postharvest pigeon pea [Cajanus cajan (L.) Millsp.] leaves. J Agric Food Chem 2013; 61: 1165-1171.

41 Colquhoun TA, Schwieterman ML, Gilbert JL et al. Light modulation of volatile organic compounds from petunia flowers and select fruits. Postharvest Biol Technol 2013; 86: 37-44.

42 Sakalauskaite J, Viskelis $\mathrm{P}$, Dambrauskienè E et al. The effects of different UV-B radiation intensities on morphological and biochemical characteristics in Ocimum basilicum L. J Sci Food Agric 2013; 93: 1266-1271.

43 Cartea M, Velasco P. Glucosinolates in Brassica foods: bioavailability in food and significance for human health. Phytochem Rev 2008; 7: 213-229.

44 Hasegawa T, Yamada K, Kosemura S, Yamamura S, Hasegawa K. Phototropic stimulation induces the conversion of glucosinolate to phototropism-regulating substances of radish hypocotyls. Phytochemistry 2000; 54: 275-279.

45 Fahey JW, Zalcmann AT, Talalay P. The chemical diversity and distribution of glucosinolates and isothiocyanates among plants. Phytochemistry 2001; 56: $5-51$. 
46 Velasco P, Soengas P, Vilar M, Cartea ME, del Rio M. Comparison of glucosinolate profiles in leaf and seed tissues of different Brassica napus crops. J Am Soc Hort SCi 2008; 133: 551-558.

47 Brown PD, Tokuhisa JG, Reichelt M, Gershenzon J. Variation of glucosinolate accumulation among different organs and developmental stages of Arabidopsis thaliana. Phytochemistry 2003; 62: 471-481.
(4) $\Theta$ This work is licensed under a Creative Commons Attribution(c) ${ }_{\mathrm{Br}} \mathrm{NC}$ ND NonCommercial-NoDerivative Works 3.0 License. To view a copy of this license, visit http://creativecommons.org/licenses/by-nc-nd/3.0/ 